\title{
Gamma Oscillatory Activity Related to Language Prediction
}

\author{
Lin Wang ${ }^{1,2,3}$, Peter Hagoort ${ }^{1,2}$, and Ole Jensen ${ }^{4}$
}

\begin{abstract}
Using magnetoencephalography, the current study examined gamma activity associated with language prediction. Participants read high- and low-constraining sentences in which the final word of the sentence was either expected or unexpected. Although no consistent gamma power difference induced by the sentencefinal words was found between the expected and unexpected conditions, the correlation of gamma power during the prediction and activation intervals of the sentence-final words was larger when
\end{abstract}

the presented words matched with the prediction compared with when the prediction was violated or when no prediction was available. This suggests that gamma magnitude relates to the match between predicted and perceived words. Moreover, the expected words induced activity with a slower gamma frequency compared with that induced by unexpected words. Overall, the current study establishes that prediction is related to gamma power correlations and a slowing of the gamma frequency.

\section{INTRODUCTION}

Language processing is predictive in the sense that context influences the state of the language processing system before the actual word input (Kuperberg \& Jaeger, 2016). EEG and magnetoencephalography (MEG) techniques are ideal for studying prediction as they can capture the rapid change of brain states. A number of ERP/field studies have shown that comprehenders anticipate different aspects of upcoming information, such that the violation of the prediction elicits detectable brain responses (e.g., Molinaro, Barraza, \& Carreiras, 2013; DeLong, Urbach, \& Kutas, 2005; Van Berkum, Brown, Zwitserlood, Kooijman, \& Hagoort, 2005). Recently, several studies measured the ERPs during the anticipation period preceding the word input. They found that highly constraining (HC) contexts produced larger negativities compared with less constraining contexts (Freunberger \& Roehm, 2017; Grisoni, Miller, \& Pulvermüller, 2017; León-Cabrera, Rodríguez-Fornells, \& Morís, 2017; Maess, Mamashli, Obleser, Helle, \& Friederici, 2016).

The aforementioned studies focused on evoked responses, which mainly reflect stimulus-locked brain activity. However, a part of the event-induced activity is not stimulus-locked to a certain event, for example, oscillatory activity, which is not phase-aligned by the event. Neural oscillations are thought to play a crucial role in linking spa-

This paper is part of a Special Focus deriving from a symposium at the 2017 annual meeting of Cognitive Neuroscience Society, entitled "Top-Down Functions of Neural Oscillations for Speech and Language Processing"

${ }^{1}$ Max Planck Institute for Psycholinguistics, Nijmegen, the Netherlands, ${ }^{2}$ Donders Institute for Brain, Cognition, and Behaviour, Radboud University Nijmegen, the Netherlands, ${ }^{3}$ Harvard Medical School, ${ }^{4}$ University of Birmingham, UK

tially distributed representations and functionally related brain regions (Fries, 2005; Engel, Fries, \& Singer, 2001; Varela, Lachaux, Rodriguez, \& Martinerie, 2001). Both slow $(<30 \mathrm{~Hz})$ and fast $(>30 \mathrm{~Hz})$ oscillatory activities have been reported during language prediction. For instance, increased theta power (Rommers, Dickson, Norton, Wlotko, \& Federmeier, 2017) and decreased beta power (Wang, Jensen, et al., 2012) have been reported when predictions were violated. Moreover, several studies found that $\mathrm{HC}$ contexts induced a theta power increase (Piai et al., 2016; Dikker \& Pylkkänen, 2013) and an alpha/beta power suppression relative to less constraining contexts (Rommers et al., 2017; Wang, Hagoort, \& Jensen, 2018; Piai, Roelofs, Rommers, \& Maris, 2015; Piai, Roelofs, \& Maris, 2014) during the anticipatory time window. These results indicate that language prediction triggers the engagement of a large-scale language network. Moreover, we found that the frontal gamma and temporal lobe alpha oscillations correlated negatively when the prediction was strong, indicating a functional connectivity between different nodes in the language network (Wang et al., 2018).

Gamma activity $(>30 \mathrm{~Hz}$ ) has been reported in response to visual or auditory word presentations. For instance, increased gamma power (around $40 \mathrm{~Hz}$ ) was observed for expected words (Monsalve, Pérez, \& Molinaro, 2014; Rommers, Dijkstra, \& Bastiaansen, 2013; Peña \& Melloni, 2012; Wang, Zhu, \& Bastiaansen, 2012; Penolazzi, Angrilli, \& Job, 2009; Hald, Bastiaansen, \& Hagoort, 2006) but not for unexpected words (but see Hagoort, Hald, Bastiaansen, \& Petersson, 2004). Moreover, increased long-range gammaphase synchronization was found for high- compared with low-constraining (LC) contexts both before and after a target word was presented (Molinaro et al., 2013). Therefore, increased gamma activity has been suggested to 
reflect the match between the received linguistic input and the preactivated lexical representations (Lewis, Wang, \& Bastiaansen, 2015). This notion is consistent with the view that synchronization in the gamma band plays a role in binding together information from external sensory input and internal top-down processes (Tallon-Baudry \& Bertrand, 1999). For instance, increased gamma power was found for stimuli that matched with the representations stored in long-term and working memory (Osipova et al., 2006; Herrmann, Lenz, Junge, Busch, \& Maess, 2004; Herrmann \& Mecklinger, 2001), indicating that the successful matching between external input and internal representation induces gamma power increase.

However, associating increased gamma power to confirmed predictions is in contradiction to the proposal that gamma activity reflects prediction error (Friston, Bastos, Pinotsis, \& Litvak, 2015; Arnal \& Giraud, 2012) in the context of the predictive coding framework (Clark, 2013; Friston, 2011; Rao \& Ballard, 1999). According to this framework, the brain infers the possible causes of sensory input based on prior experiences. These generated hypotheses are then compared with incoming sensory information. Prediction error reflects the difference between the top-down expectation and incoming sensory inputs. In the case where no strong prediction of upcoming input is available, the bottom-up input is unpredicted, and thus, a prediction error will be generated as well. The prediction error is propagated forward throughout the cortical hierarchy via gamma activity, with unexpected stimuli producing greater gamma power. Supporting evidence primarily comes from visual (Bastos et al., 2015) and auditory (Arnal, Wyart, \& Giraud, 2011; Todorovic, van Ede, Maris, \& de Lange, 2011) perception studies, but experimental evidence from higher-order cognitive domains remains elusive.

In the current study, we presented sentences with HC or LC contexts. At the same time, the sentence-final word (SFW) was either congruent (C) or incongruent (IC) relative to the context. Consequently, the SFWs were expected in the high-constraining and congruent $(\mathrm{HC} / \mathrm{C})$ condition but not in the other three conditions: high-constraining incongruent $(\mathrm{HC} / \mathrm{IC})$, low-constraining congruent $(\mathrm{LC} / \mathrm{C})$, low-constraining incongruent (LC/IC). Note that the same data set was analyzed in our previously published study (Wang et al., 2018). However, this study focused on the gamma activity associated with prediction using very different approaches. The first aim of the current study was to examine the gamma power induced by the expected (the C SFWs in the HC contexts) and unexpected (the IC SFWs in the HC contexts as well as the SFWs in the LC contexts) words. If gamma power relates to the agreement between the preactivated words and the words that are actually presented, the gamma power induced by the expected words in the HC contexts should be higher than the power induced by the unexpected words in the other three conditions. On the contrary, if gamma power relates to prediction error (i.e., the mismatch between prediction and bottom-up input), the gamma power should be higher for the unexpected words in both the HC and LC contexts than the expected words in the HC context.

In addition to examining the gamma power induced by the sentence-final words (SFWs), we were also interested in how the gamma activity in response to the presented words related to the gamma activity associated with the prediction of those words. In the working memory literature, it has been shown that the spatial/temporal pattern of brain activity during encoding and retrieval of remembered items is highly similar (e.g., Wolff, Jochim, Akyurek, \& Stokes, 2017; Michelmann, Bowman, \& Hanslmayr, 2016; Staudigl, Vollmar, Noachtar, \& Hanslmayr, 2015) and that content-specific information can be decoded from gamma activity (Zhang et al., 2015; Polanía, Paulus, \& Nitsche, 2012). By correlating the gamma-band activity between the activation and prediction intervals across trials, we would be able to further test whether the magnitude of the gamma activity relates to item-specific predictions. We hypothesized that a word associated with high gamma power in the activation time window will induce high gamma power in the prediction time window within the same trial. Therefore, if gamma activity indeed reflects the match between predicted and perceived words, the correlation of the gamma power between the activation and prediction periods should be greater in the $\mathrm{HC} / \mathrm{C}$ condition than the HC/IC conditions or the LC conditions, as the item-specific preactivation is only made and matched in the $\mathrm{HC} / \mathrm{C}$ condition.

Finally, we quantified the frequency content of the gamma activity induced by the expected (in the HC/C condition) and unexpected (in the other three conditions) words to test whether there was a change in gamma frequency. Slower and faster gamma activities have been associated with prospective memory retrieval and maintenance of recent sensory information respectively in rat hippocampal recordings (Colgin \& Moser, 2010). In the current study, we expected a slower gamma frequency for the expected words in the $\mathrm{HC} / \mathrm{C}$ condition (as the highly predictive contexts could facilitate prospective retrieval) and a faster gamma frequency for the unexpected words in the other three conditions (because they might be maintained temporarily to be integrated into the contexts).

\section{METHODS}

The participants, stimuli, procedure, and data acquisition have been reported more extensively in Wang et al. (2018).

\section{Participants}

Thirty-four right-handed native Dutch speakers (mean age $=$ 24 years, range $=20-35$ years, 13 men) served as paid volunteers. They had normal or corrected-to-normal vision. None of them had dyslexia or any neurological impairment. They signed a written consent form according to the Declaration of Helsinki. The data of one man 
and one woman were excluded because of severe metalrelated artifacts from dental work. The final set of participants therefore consisted of 32 participants (mean age $=24$ years, range $=20-35$ years, 12 men) .

\section{Stimulus}

We constructed 240 Dutch sentence pairs, each pair ending with the same SFW (see Table 1 for some examples). Each sentence pair differed in only one word, which preceded the SFW by at least two words. The differing words in each sentence pair created either HC or LC contexts, so that the SFW could be predicted in the HC context whereas it could not be predicted in the LC context. A cloze probability test was conducted to quantify the sentence constraints in two groups of participants who did not participate in the MEG study. The semantic constraint of the context was quantified by the percentage of participants who filled in the most common word for each sentence. The cloze test showed that the HC sentences had higher contextual constraints than the LC sentences: mean $(S D)=86 \%(11 \%)$ and $28 \%(10 \%)$, respectively; $t(478)=62.27, p<.001$. The cloze probability of the SFW was quantified by the percentage of the participants who completed the sentence with that word. The SFW had higher cloze probability in the HC sentences (86\%) than in the LC sentences (6\%). The mean sentence length was eight words (range $=5-15$ words).

We also manipulated the semantic congruence of the SFWs by replacing the expected words with words that

Table 1. Examples of Two Items in Four Conditions

1. HC/LC, C/IC

HC-C/IC: Hij gaf haar een ketting voor haar verjaardag/borstel

(He gave her a necklace for her birthday/brush.)

LC-C/IC: Hij gaf haar een ticket voor haar verjaardag/borstel.

(He gave her a ticket for her birthday/brush.)

\section{HC/LC, C/IC}

HC-C/IC: Om de cellen te kunnen zien gebruikte hij een $\underline{\text { microscoop/kathedraal. }}$

(In order to see the cells he used a microscope/cathedral.)

LC-C/IC: Om de objecten te kunnen zien gebruikte hij een $\underline{\text { microscoop } / \text { kathedraal. }}$

(In order to see the objects he used a microscope/cathedral.)

Statement: Hij gebruikte een apparaat om iets te kunnen zien.

(He used a device in order to see something.)

The examples were originally in Dutch, with the SFWs underlined. The critical words that create different contextual constraints were in bold The target words were underlined. The English translations are given in brackets below the original Dutch materials. An example of the statement (which required YES answer) was provided for Example 2. made the sentences incongruent in both the $\mathrm{HC}$ and LC contexts. A sentence plausibility test was conducted to quantify the semantic congruency in a different group of participants. They were asked to rate the plausibility of each sentence on a scale from 1 (bighly implausible) to 7 (bighly plausible). The C sentences were rated to be more plausible than the IC sentences. Also, the plausibility difference between the IC and $\mathrm{C}$ sentences was larger for the HC than for the LC sentences. The mean and $S D$ of the ratings in the four conditions were as follows: $\mathrm{HC} / \mathrm{C}$ : 6.49 (0.09), HC/IC: 1.59 (0.10), LC/C: 5.79 (0.12), LC/IC: 1.94 (0.12). Moreover, the IC and C words were matched on word category, animacy, word frequency, and word length. The four conditions of all 240 sentences were distributed among four lists with a Latin square design, so that each participant read 60 sentences of the same condition.

\section{Procedure}

Participants were tested individually in a magnetically shielded room. They were seated in a comfortable chair under the MEG helmet, facing a projected screen at approximately $80-\mathrm{cm}$ distance. The stimuli were presented in gray color on a black background on the screen, with a font size of 36 for the words and 30 for the probe statements. A trial started with a blank screen (duration $1600 \mathrm{msec}$ ), followed by a sentence that was presented word by word. Each word was presented for $200 \mathrm{msec}$, with an ISI of $800 \mathrm{msec}$. The last word ended with a period. After $1600 \mathrm{msec}$, the participants either saw a statement (20\% of trials) or a "NEXT" signal. For the trials in which participants saw a statement following the sentence, they were required to judge the accuracy of the statement by pressing one of two buttons to ensure that they had read for comprehension. In the other trials, the participants were instructed to press a third button. All responses were required to be delivered within $5000 \mathrm{msec}$. After a response, the next trial began. Participants were asked not to move or blink when individual words appeared, but they were encouraged to blink during the presentation of the questions.

Participants read one list of 240 sentences in a pseudorandom order. No more than three sentences of the same condition were presented in succession. The 240 sentences in one list were divided into 12 blocks ( 24 trials per block), with each block lasting about $5 \mathrm{~min}$. Between each block, there was a small break, after which participants could start the next block by informing the experimenter. The whole experiment took about $1.5 \mathrm{hr}$, including participants' preparation, instructions, and a short practice session consisting of 12 sentences.

\section{Data Acquisition}

MEG signals were recorded with 275 axial gradiometers CTF Omega System. In addition, horizontal and vertical EOG as well as electrocardiography were recorded to later discard trials contaminated by eye movements, blinks, 
and heart beats. The ongoing MEG and EOG signals were low-pass filtered at $300 \mathrm{~Hz}$, digitized at $1200 \mathrm{~Hz}$, and stored for offline analysis. To measure the head position with respect to the axial gradiometers, three coils were placed at anatomical landmarks of the head (nasion, left and right ear canal). Head position was monitored in real time (Stolk, Todorovic, Schoffelen, \& Oostenveld, 2013).

\section{Data Preprocessing}

Data were analyzed using the Fieldtrip software package, an open-source MATLAB toolbox (Oostenveld, Fries, Maris, $\&$ Schoffelen, 2011). We analyzed the time window of -2 to $2 \mathrm{sec}$ relative to the SFWs (including $2 \mathrm{sec}$ after the SFW as well as the two immediately preceding words, i.e., SFW-1 and SFW-2). A third-order synthetic gradiometer correction was applied to remove noise from the environment. Trials contaminated with muscle or MEG jump artifacts were identified and removed using a semiautomatic routine. After that, we performed independent component analysis (Jung et al., 2000; Bell \& Sejnowski, 1997) to the data and removed independent component analysis components associated with eye movement and cardiac-related activities from the MEG signals. Ultimately, we inspected the data visually and removed any remaining artifacts. In the end, on average $96 \%$ of trials were kept, with equal numbers of trials (58 trials on average) among the four conditions (all $p s>.19$ ).

\section{Time-Frequency Representations of Gamma Power}

The time-frequency representations (TFRs) of the single trials were calculated in the frequency range of $30-200 \mathrm{~Hz}$ using a multitaper approach (Mitra \& Pesaran, 1999). Power estimates were computed with a 200-msec time-smoothing and a $10-\mathrm{Hz}$ frequency-smoothing window, in $5-\mathrm{Hz}$ frequency steps and 50-msec time steps. The TFRs were calculated at each sensor location for the vertical and horizontal planar gradient and then combined (Bastiaansen \& Knösche, 2000). The planar gradient TFRs of the HC and LC conditions were averaged separately for each participant. The TFRs were $\log _{10}$-transformed and the power changes in the poststimulus interval were expressed as an absolute change from the -1750 to $-1250 \mathrm{msec}$ baseline prestimulus interval (i.e., $\log _{10}\left(\right.$ Power $_{\text {post }} /$ Power $\left._{\text {pre }}\right)$. The baseline correction was conducted to visualize the induced gamma power (Figure 1). No significant difference was found in the gamma power in the baseline period. Because of temporal smearing, any given time point in the resulting TFR is a weighted average of the time window of $\pm 100 \mathrm{msec}$.

\section{TFRs of $\mathrm{R}$ values for the Correlation between Pre- and Post-SFW Gamma Power}

To examine whether the gamma activity is associated with representational-specific preactivations, we correlated the gamma power induced by the SFWs (i.e., activation amplitude) with the gamma power associated with the prediction of the SFWs (i.e., prediction amplitude) across trials before baseline correction. We focused on the early stage of word encoding, such as visual word-form analysis (Leonard et al., 2013; Hauk, Davis, Ford, Pulvermüller, \& Marslen-Wilson, 2006), such that the activation was estimated within the first $200 \mathrm{msec}$. This was done to isolate the brain activity that reflects the initial analysis of the presented words instead of the integration of the words with previous contexts (Kutas \& Federmeier, 2011). If gamma activity is associated with representational-specific preactivations, the activation amplitude should more closely resemble the prediction amplitude when there was a strong prediction (i.e., HC/C) compared with when there was no clear prediction (i.e., $\mathrm{LC} / \mathrm{C}$ and $\mathrm{LC} / \mathrm{IC}$ ) of upcoming words or a violation of the prediction (i.e., $\mathrm{HC} / \mathrm{IC}$ ). As shown in Figure 2A, we first calculated the TFR of gamma power for each trial (as described in Time-Frequency Representations of Gamma Power). The gamma power values at the $100-\mathrm{msec}$ time point (i.e., the weighted average of the gamma power in the 0-200 msec time window) reflected the activation amplitude in response to the SFWs. Likewise, the gamma power values between the -800 and -200 msec time window related to the prediction amplitude associated with the SFWs. We calculated Spearman correlations between the activation amplitude at 100 msec and the prediction amplitude at each time point in the -800 to $-200 \mathrm{msec}$ time window and each frequency point in the $50-100 \mathrm{~Hz}$ frequency band across trials, for each sensor and each participant. This resulted in a TFR of $R$ values for each sensor and each participant. We conducted this analysis separately for the trials of the four conditions. The TFR of $R$ values in the four conditions, as well as the difference between the HC/C and each of the HC/IC, LC/C, LC/IC conditions, are shown in Figure 2B. The topographic distributions of the $R$ values in selected time and frequency windows (see Results) are shown in Figure 2C.

\section{Measure the Gamma-dominating Frequency by Calculating the Center Frequency of Power}

After establishing a link between gamma activity and representational-specific preactivation, we further tested whether the frequency of the gamma activity differed between the four conditions. The frequency was quantified as center of power in the $50-100 \mathrm{~Hz}$ frequency range. We first estimated the gamma power spectrum by averaging the trial-averaged TFRs over time (100-350 msec relative to the SFWs). Then the center frequency of power

$(\mathrm{CFOP})$ was calculated as $\mathrm{CFoP}=\frac{\sum_{k=1}^{n} k \times \operatorname{power}(k)}{\sum_{k=1}^{n} \operatorname{power}(k)}$, where

$k$ represents the frequency and power $(k)$ represents the power at frequency $k$. This gave us an estimation of 
Figure 1. TFRs of gamma power in four conditions at one left posterior sensor (MLO42), with relative power change compared with the baseline period $(-1.75$ to $-1.25 \mathrm{sec}$, not shown). The SFW started at $0 \mathrm{sec}$. The presentation of words $(-1$ to $-0.8 \mathrm{sec}$ and $0-0.2 \mathrm{sec})$ induced gamma power increase in the $0.1-0.35 \mathrm{sec}$ time window relative to words' onsets. The gamma power showed strong posterior distribution, as shown in the topographic plots under the TFR plots. No significant gamma power difference was found between the $\mathrm{HC} / \mathrm{C}$ and $\mathrm{LC} / \mathrm{C}$ or $\mathrm{LC} / \mathrm{IC}$ conditions in either the pre-SFWs or post-SFWs time interval. The gamma power was stronger in the $\mathrm{HC} / \mathrm{IC}$ than the $\mathrm{HC} / \mathrm{C}$ condition in the 300-600 msec interval relative to the onset of $\mathrm{SFW}$ over left temporal sensors.

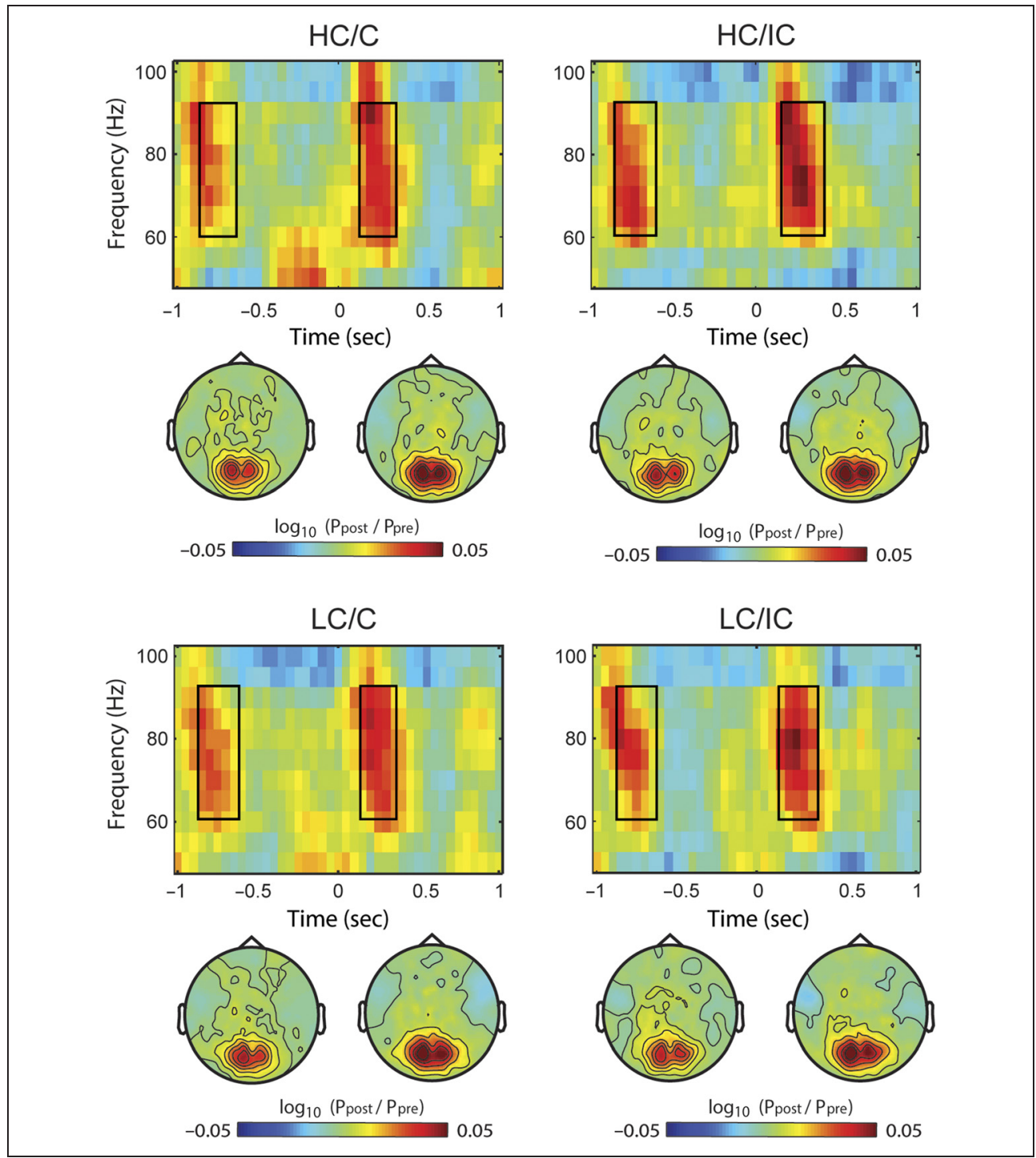

the dominating frequency within $50-100 \mathrm{~Hz}$ in the time window of 100-350 msec relative to the SFWs. We calculated the CFoP for six posterior sensors where the representational-specific gamma activity was most prominent (as circled in the topographic map in Figure 2C). This was done separately for the four conditions for each participant. We compared the $\mathrm{HC} / \mathrm{C}$ condition with the other three conditions, as the prediction was only confirmed in the $\mathrm{HC} / \mathrm{C}$ condition whereas it was violated in the $\mathrm{HC} / \mathrm{IC}$ condition and it was unpredictable in the LC conditions. To test whether the center frequency difference could be exclusively explained by the predictability of the SFWs, we also calculated the CFoP of the gamma activity induced by the pre-SFW between 100 and $350 \mathrm{msec}$ after the SFW-1 was presented. The CFoP difference between conditions was statistically tested using ANOVA on the CFoP values over six posterior sensors, with two withinsubject factors of Conditions (HC/C, HC/IC, LC/C, LC/IC) and Time windows (post-SFW, pre-SFW). GreenhouseGeisser correction was applied when the degree of free- dom in the numerator was larger than one. The original degrees of freedom with corrected $p$ values were reported.

\section{Cluster-based Permutation Statistics}

We performed cluster-based permutation tests (Maris \& Oostenveld, 2007) across participants for the TFR of power and the TFR of $R$ values. Based on previous MEG studies (Arnal et al., 2011; Todorovic et al., 2011) as well as visual inspection of the data, we statistically quantified the gamma power difference (Figure 1) in the 60-90 Hz frequency band between the four conditions both before ( $-1000-0 \mathrm{msec})$ and after $(0-1000 \mathrm{msec})$ the presentation of the SFWs. As for the TFR of $R$ values (Figure 2), we compared the $R$ values within 60-90 Hz frequency bands in the prediction period $(-800$ to $-200 \mathrm{msec})$ relative to SFWs to avoid any contamination of the evoked responses to the pre-SFWs (which were presented during -1000 to -800 msec relative to SFWs). All sensors and 


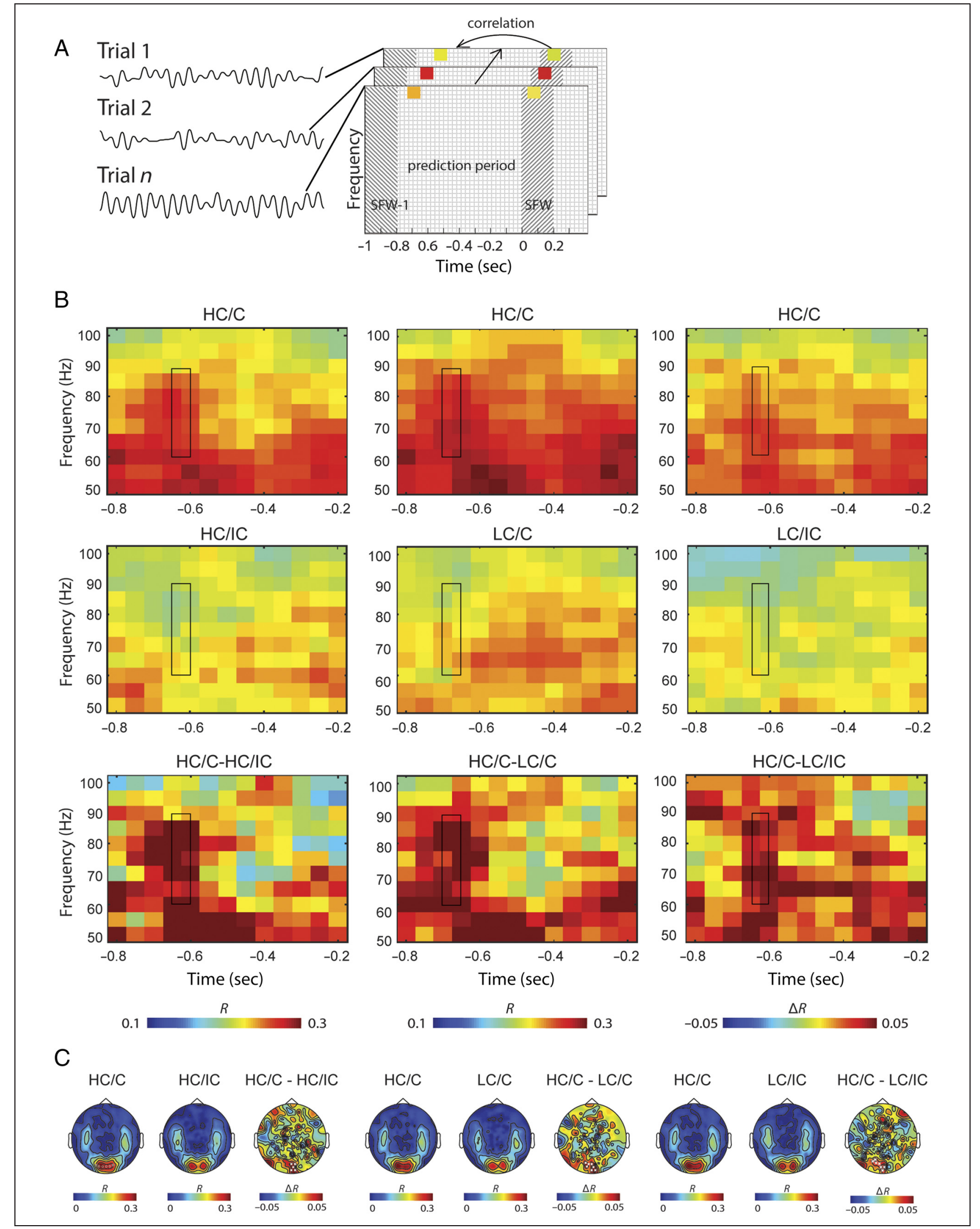


time points were included into the permutation test initially. After identifying the time windows that showed significant effects, we averaged the data within the time interval, and then the cluster was defined at the sensor level. A brief description of the cluster-based permutation test is as follows. First, for each data sample of the observed data (i.e., sensor or sensor by time data sample), we computed the mean difference between two conditions. Clusters were defined by the 95th percentile of the mean difference values, and the sum of the mean difference values within each cluster was calculated. Next, a null distribution was created by randomly assigning the values to the two conditions 1000 times, with the largest cluster-level statistic in each permutation entering the null distribution. Finally, each observed cluster-level statistic was compared with the permutation distribution to assess significance for each cluster. Clusters falling in the highest or lowest 2.5 th percentile were considered significant.

\section{RESULTS}

Participants read HC or LC sentences that ended with either $\mathrm{C}$ or IC words. They were asked to judge the correctness of statements in $20 \%$ of the sentences. Participants made highly accurate responses in all conditions $($ mean $(S D)=98.7 \%(0.2 \%), 98.3 \%(0.3 \%), 98.3 \%$ $(0.3 \%)$, and $97.6 \%(0.3 \%)$, respectively, for the $\mathrm{HC} / \mathrm{C}$, $\mathrm{HC} / \mathrm{IC}, \mathrm{LC} / \mathrm{C}$, and LC/IC conditions), suggesting that they carefully read the sentences for comprehension. The accuracy was slightly higher in the $\mathrm{C}$ than the $\mathrm{IC}$ condition, as indicated by a main effect of Congruency: $F(1,31)=4.394$, $p=.044, \eta^{2}=.124$. No difference was found in the response time: mean $(S D)=1318 \mathrm{msec}(96 \mathrm{msec}), 1330 \mathrm{msec}$ (102 msec), $1298 \mathrm{msec}$ ( $96 \mathrm{msec}$ ), and $1334 \mathrm{msec}$ (102 msec), respectively, for the $\mathrm{HC} / \mathrm{C}, \mathrm{HC} / \mathrm{IC}, \mathrm{LC} / \mathrm{C}$, and $\mathrm{LC} / \mathrm{IC}$ conditions, all $p s>.150$.

The low-frequency power effects as well as the source localization results can be found in Wang et al. (2018). Figure 1A shows the gamma power induced by the SFWs and prefinal words (SFWs-1) in the four conditions after a baseline correction of -1750 to $-1250 \mathrm{msec}$ (i.e., preceding SFW-1). The visual presentation of words induced gamma power increase in the 100-350 msec time window after the words' onsets. A cluster-based permutation test conducted on the averaged gamma power in the 60$90 \mathrm{~Hz}$ across the 0-1000 msec interval revealed reduced gamma power in the $\mathrm{HC} / \mathrm{C}$ than the $\mathrm{HC} / \mathrm{IC}$ condition between 300 and $600 \mathrm{msec}(p=.026)$. However, no gamma difference was found between $\mathrm{HC} / \mathrm{C}$ and $\mathrm{LC} / \mathrm{C}$ ( $p=.252$ ) or between HC/C and LC/IC ( $p=1.0)$ conditions. The results suggest that the gamma power induced by the expected words in the HC contexts did not differ from the unexpected words in the LC contexts. In addition, no gamma power difference was found before the SFWs were presented (all ps > .60), suggesting that the gamma power difference was not sensitive to the prediction difference.

Previous studies have shown that pattern of gamma activity relates to item-specific representations (Zhang et al., 2015; Polanía et al., 2012), so it is very likely that the gamma activity associated with the activation of a specific word resembles the prediction of that word. To test this, we correlated the gamma power induced by the SFWs with the gamma power during the prediction period where no words were presented (i.e., -800 to -200 msec relative to SFWs). We hypothesized that if gamma activity relates to representational-specific prediction, the gamma power correlation between the activation period (around $100 \mathrm{msec}$ ) and the prediction period ( -800 to $-200 \mathrm{msec}$ relative to the SFWs) should be stronger in the $\mathrm{HC} / \mathrm{C}$ than in the other conditions. The cluster-based permutation test conducted to the -800 to -200 msec interval revealed a larger correlation in the gamma frequency band (60-90 Hz) for the $\mathrm{HC} / \mathrm{C}$ condition compared with the HC/IC $(p=.022), \mathrm{LC} / \mathrm{C}$ $(p=.008)$, as well as LC/IC conditions $(p=.022)$. Then, we identified the time interval that showed significant effect (Figure 2B) and averaged the $R$ values within the time interval for cluster-based permutation test at the sensor level to identify the sensors that showed significant effects (Figure 2C). The larger correlation for the $\mathrm{HC} / \mathrm{C}$ condition was found over posterior sensors (as marked by white asterisks in Figure 2C) in the time interval of -650 to $-600 \mathrm{msec},-700$ to $-650 \mathrm{msec}$, and -650 to $-600 \mathrm{msec}$, respectively, for the $\mathrm{HC} / \mathrm{IC}(p=.002), \mathrm{LC} / \mathrm{C}$ $(p=.002)$, and LC/IC ( $p=.004)$ conditions. The highly significant effects indicate that the correlation difference between the $\mathrm{HC} / \mathrm{C}$ and the other conditions was very robust. Because previous studies have also reported prediction modulation on the power of low-frequency bands (2-30 Hz; Rommers et al., 2017; Wang et al., 2018; Piai et al., 2014, 2015, 2016; Dikker \& Pylkkänen, 2013), we

\footnotetext{
Figure 2. Correlation between induced gamma power during the pre- and post-SFWs time windows. (A) An illustration of the correlation analysis. First, the time series of all trials were transformed to time-frequency domains. The gamma power in the post-SFW interval at $100 \mathrm{msec}$ (the weighted gamma power in the time window of $0-0.2 \mathrm{sec}$ ) reflected the activations in response to the SFWs, which served as reference gamma activity. The reference gamma activity (at $0.1 \mathrm{sec}$ ) was correlated with the gamma power values at each time point (in the -0.8 to $-0.2 \mathrm{sec}$ time window relative to the SFWs) and frequency data point (50-100 Hz) across trials. This resulted in a TFR of $R$ values for each sensor and each participant. The analysis was conducted separately for the trials in the $\mathrm{HC} / \mathrm{C}, \mathrm{HC} / \mathrm{IC}, \mathrm{LC} / \mathrm{C}$, and $\mathrm{LC} / \mathrm{IC}$ conditions. (B) TFR of $R$ values in the four conditions as well as three contrasts: HC/C versus HC/IC (left), HC/C versus LC/C (middle), HC/C versus LC/IC (right). The $R$ values were averaged over sensors that showed significant difference for each contrast. Between 60 and $90 \mathrm{~Hz}$ frequency, the correlation was stronger in the HC/C than the HC/IC, $\mathrm{LC} / \mathrm{C}$, and LC/IC conditions during -0.65 to $-0.6 \mathrm{sec},-0.7$ to $-0.65 \mathrm{sec}$, and -0.65 to $-0.6 \mathrm{sec}$ time windows, respectively. (C) The topographic distributions of the $R$ values in the frequency and time intervals that showed significant effects for the four conditions as well as the contrasting conditions. The sensors showing significant effects were marked by white asterisks.
} 
Figure 3. Averaged gamma power spectrum in the $0.1-0.45$ sec time window over six posterior sensors (as circled in the first topographical map in Figure 2C). (A) Gamma power spectrum averaged in the $0.1-0.35$ sec time window relative to the SFWs. The dominating gamma frequency in the $\mathrm{HC} / \mathrm{C}$ condition was lower than that in the $\mathrm{HC} / \mathrm{IC}$, LC/C, and LC/IC conditions. (B) Scatter plot of the CFoP value in the $\mathrm{HC} / \mathrm{C}$ condition versus that in the $\mathrm{HC} / \mathrm{IC}$ (in red dot), LC/C (in blue dot), and LC/IC (in green dot) conditions in the post-SFW interval for 32 participants. Most dots fall above the diagonal line, indicating that the CFoP values in the $\mathrm{HC} / \mathrm{C}$ condition were smaller than those in the other conditions for most participants. (C) Gamma power spectrum in the $0.1-0.35$ sec time window relative to the pre-SFWs. The dominating gamma frequency in the four conditions showed no statistically significant difference. (D) Scatter plot of the CFoP value in the HC/C condition versus that in the HC/IC (in red dot), LC/C (in blue dot), and LC/IC (in green dot) conditions in the pre-SFWs interval for 32 participants. The number of dots above and below the diagonal line was similar, suggesting no clear $\mathrm{CFoP}$ values difference between the $\mathrm{HC} / \mathrm{C}$ and the other three conditions.
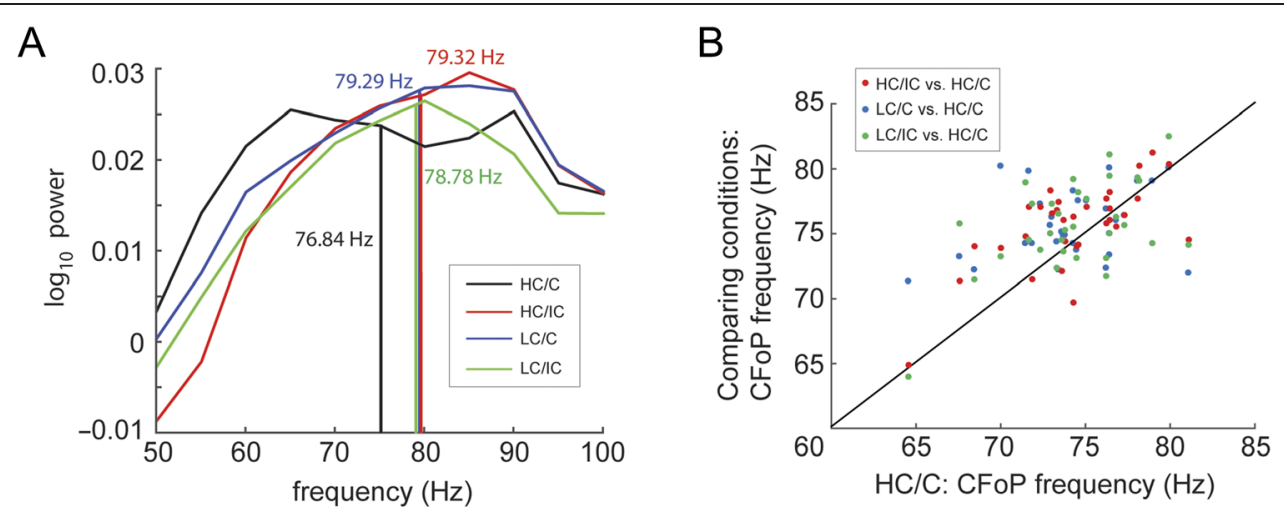

C

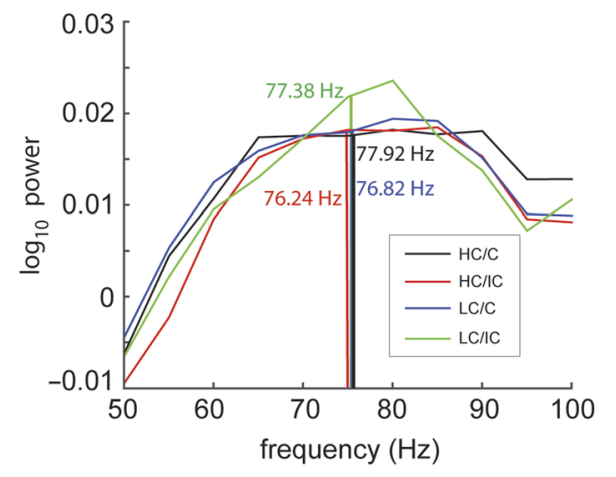

D

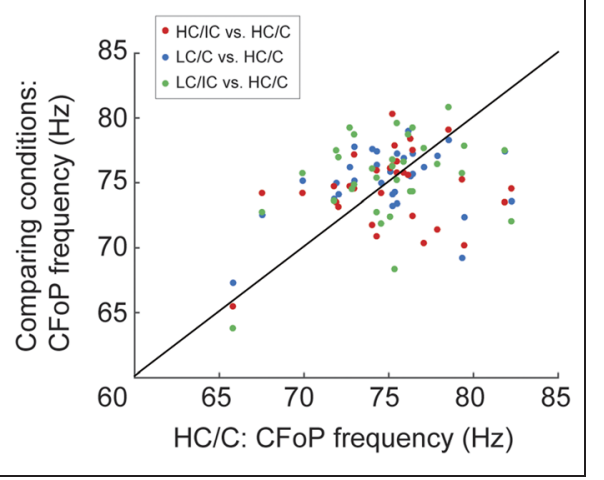

also conducted a similar correlation analysis to the lowfrequency band. The statistical test between the $\mathrm{HC} / \mathrm{C}$ condition and the other conditions (i.e., HC/IC, LC/C, LC/LC) did not yield any significant effect in the theta (3-8 Hz), alpha (8-12 Hz), or beta $(15-20 \mathrm{~Hz})$ frequency band, indicating that the correlation effect was specific to the gamma frequency band.

Previous studies based on place recordings in the rat have shown that slow and fast frequencies of hippocampal gamma activity relate to prospective spatial representations retrieved from memory and retrospective spatial representations reflecting the immediate past, respectively. In the current study, the HC context may have triggered prospective retrieval, which would predict more gamma power to be present at slower frequencies when comparing the $\mathrm{HC} / \mathrm{C}$ to the other conditions. Indeed, using a CFoP analysis, we found that the center frequency of the gamma activity was lower in the $\mathrm{HC} / \mathrm{C}$ than the $\mathrm{HC} / \mathrm{IC}, t(31)=2.72, p=.010, \mathrm{LC} / \mathrm{C}, t(31)=2.56, p=$ .016 , and $\mathrm{LC} / \mathrm{IC}, t(31)=2.32, p=.027$, conditions: $F(3$, $93)=3.382, p=.026, \eta^{2}=.098$ (Figure $3 \mathrm{~A}$ ). The scatter plot of the CFoP of the $\mathrm{HC} / \mathrm{C}$ condition against the other three comparing conditions confirmed this observation (Figure 3B), showing that most participants had a slower dominating gamma frequency in the $\mathrm{HC} / \mathrm{C}$ than the comparing conditions (points above the diagonal line). To test whether this was solely due to the predictability of the SFWs, we also compared the CFoP of the gamma activity in the pre-SFWs interval between the HC/C and each of other conditions. The gamma activity in the pre-SFW interval showed no CFoP difference between $\mathrm{HC} / \mathrm{C}$ and $\mathrm{HC} / \mathrm{C}$, LC/C, or LC/IC conditions: $F(3,93)=.612, p=.593, \eta^{2}=$ .019 , as shown in Figure $3 \mathrm{C}$ and $\mathrm{D}$. The interaction test between conditions (HC/C, HC/IC, LC/C, LC/IC) and time window (SFW, pre-SFW) showed a marginally significant effect: $F(3,93)=2.570, p=.068, \eta^{2}=.077$.

\section{DISCUSSION}

The current study examined gamma activity associated with language prediction when participants read sentences ending with expected words in $\mathrm{HC}$ context $(\mathrm{HC} / \mathrm{C})$ or unexpected words in $\mathrm{HC}$ context (HC/IC) or unexpected words in LC contexts (LC/C and LC/IC). No consistent difference in gamma power was found between the $\mathrm{HC} / \mathrm{C}$ and other conditions in either the prediction or activation time windows. However, the gamma power in the prediction and activation time windows were more similar when the prediction was confirmed compared with when the prediction was violated or when no strong prediction could be made. In addition, the processing of expected words in the HC condition induced gamma activity with 
a slower frequency compared with the processing of unexpected words in the other conditions.

Unlike previous studies (Monsalve et al., 2014; Rommers et al., 2013; Wang, Zhu, et al., 2012; Penolazzi et al., 2009; Hald et al., 2006; Hagoort et al., 2004), the current study found no significant gamma power difference elicited by the expected words in the HC contexts and the unpredictable words in the LC contexts. It should be noted, however, that the violation of prediction (HC/IC vs. HC/C) produced stronger gamma activity in the HC contexts between 300 and $600 \mathrm{msec}$. In addition, the test of congruency effect $(\mathrm{HC} / \mathrm{IC}+\mathrm{LC} / \mathrm{IC}$ vs. HC/C $+\mathrm{LC} / \mathrm{C})$ revealed stronger gamma power in the IC than in the $\mathrm{C}$ conditions in the 150-650 msec interval ( $p=.024)$, as reported in our previous article (Wang et al., 2018). As discussed in Lewis and Bastiaansen (2015), the mixed findings on the gamma power difference between the expected and unexpected inputs might be explained by a potential confound between prediction and attention. It has been shown that attended stimuli trigger stronger gamma-band responses than unattended stimuli (Jensen \& Colgin, 2007; Bauer, Oostenveld, Peeters, \& Fries, 2006; Gruber, Müller, Keil, \& Elbert, 1999). Because it is difficult to disentangle prediction from attention (Summerfield \& de Lange, 2014) and various factors can affect attention (such as the proportion of violating stimuli in the experiment and the task requirement), the lack of a consistent gamma power difference in the current study might be explained by the confound of attention.

By correlating the gamma power between the activation and prediction time windows across trials, we found that the gamma activity induced by processing the expected word was similar to the gamma activity induced by the prediction of that word in the $\mathrm{HC} / \mathrm{C}$ condition. Previous studies have shown that remembered items induced gamma activity with similar spatial and temporal patterns between encoding and retrieval intervals (Zhang et al., 2015) and that specific information maintained in visual working memory can be decoded from gamma oscillatory patterns in the prefrontal cortex (Polanía et al., 2012). In the current study, gamma power in the poststimuli and prestimuli time windows was related to itemspecific activation and preactivation, respectively. We then correlated gamma power across trials separately for the $\mathrm{HC} / \mathrm{C}, \mathrm{HC} / \mathrm{IC}, \mathrm{LC} / \mathrm{C}$, and LC/IC conditions. In the HC/C condition, the same word was preactivated and processed, resulting in a high correlation of gamma power between the activation and prediction time windows. In the HC/IC condition, the perceived word differed from the predicted word, resulting in a weaker correlation. In the LC conditions, no specific word could be predicted, so the magnitude of gamma activity associated with the processing of the unexpected word did not resemble the magnitude of gamma activity during the prediction interval, also leading to a lower correlation of gamma power between the activation and prediction time windows. The effect was most robust during the -700 to $-650 \mathrm{msec}$ interval preceding the onset of the SFWs, which overlapped with the gamma activity induced by the pre-SFWs (SFW-1) in the -900 to $-650 \mathrm{msec}$ interval. It is very likely that the processing of the SFW-1 and the preactivation of the SFW co-occurred. However, because the SFW differed from the SFW-1 across all conditions, the greater gamma power correlation between the pre- and poststimuli intervals could only be attributed to the preactivation of the highly predicted words. Moreover, this gamma correlation effect was mainly found in posterior regions (Figure 2C), presumably over the visual cortex. It has been shown that people make predictions at the level of the visual/orthographic features of upcoming words whenever it is possible (Wang et al., 2018; Brothers, Swaab, \& Traxler, 2015; Molinaro et al., 2013; Kim \& Lai, 2012; Dikker \& Pylkkanen, 2011; Laszlo $\&$ Federmeier, 2011). The induced gamma activity around $100 \mathrm{msec}$ after the onset of the SFW most likely reflects the visual processing of the presented words (Leonard et al., 2013; Hauk et al., 2006). Therefore, the high correlation of gamma activity between the activation and prediction of the expected words in the HC/C condition might be due to predictions of the visual/orthographic characteristics of the lexical items.

The higher gamma power correlation between the prediction and activation periods in the $\mathrm{HC} / \mathrm{C}$ than in the $\mathrm{HC} / \mathrm{IC}$, LC/C, and LC/IC conditions seems to be consistent with the notion that gamma activity relates to the matching of preactivation and processing of predicted words (Lewis et al., 2015), rather than reflecting prediction error (Friston et al., 2015; Arnal \& Giraud, 2012). According to the predictive coding framework (Clark, 2013; Friston, 2011; Rao \& Ballard, 1999), only the prediction error (i.e., the difference between the predicted and the perceived sensory input) is propagated to higher-level cortical regions. In the current study, the expected word matched the prediction, and thus, the prediction error in the $\mathrm{HC} / \mathrm{C}$ condition was minimal. If gamma activity relates to prediction error, which reflects brain activity that cannot be explained by the prediction, then the gamma activity induced by the expected word should not correlate with the gamma activity induced during the prediction period. We found that the processing of the expected word instead showed similar gamma power to the prediction of that word. Thus, our study provides support for the notion that gamma activity relates to the match between top-down prediction and bottom-up input.

In addition, we found that the expected words in the $\mathrm{HC}$ contexts (i.e., the $\mathrm{HC} / \mathrm{C}$ condition) induced gamma activity with a slower frequency than the unexpected words in the $\mathrm{HC}$ contexts (i.e., the $\mathrm{HC} / \mathrm{IC}$ condition) as well as the words in the LC contexts (i.e., LC/C and LC/IC conditions) did. That is, although the words in all conditions induced gamma activity, the gamma activity induced in the $\mathrm{HC} / \mathrm{C}$ condition was dominated by relatively slower gamma activity compared with the other conditions. In the literature on hippocampal gamma activity, synchronization of slow gamma oscillations between CA3 
and CA1 areas has been shown to reflect prospective representations of upcoming locations whereas synchronization of fast gamma oscillations have been related to retrospective representations reflecting the immediate past (Zhang et al., 2015; Bieri, Bobbitt, \& Colgin, 2014; Colgin \& Moser, 2010; Colgin et al., 2009). In this study, the expected words in the $\mathrm{HC} / \mathrm{C}$ condition-associated with slower gamma - could be retrieved from long-term memory and represented in a predicted/prospective manner. In contrast, the unexpected words in the $\mathrm{HC} / \mathrm{IC}$ and LC conditions might be maintained temporarily in a retrospective manner to be integrated into the preceding contexts. Therefore, our finding on the relatively slower gamma in the $\mathrm{HC} / \mathrm{C}$ condition than in the unexpected conditions parallels the rat hippocampal findings.

Overall, despite the lack of consistent gamma power difference between the expected (in the $\mathrm{HC} / \mathrm{C}$ condition) and unexpected (in the LC conditions) words, the current study establishes a link between prediction and activation of highly expected words, as indicated by their strong correlation in gamma activity. In addition, it is the first study to report a lower dominating gamma frequency for expected words in the HC context compared with unexpected words in language processing, supporting a functional distinction between slow and fast gamma oscillatory activity. Therefore, it is crucial to study various aspects of gamma oscillatory activity associated with language prediction.

\section{Acknowledgments}

P. H. was supported by the NWO Spinoza Prize, the Academy Professorship Award of the Netherlands Academy of Arts and Sciences, and the NWO Language in Interaction grant; O. J. was supported by James S. McDonnell Foundation Understanding Human Cognition Collaborative Award (220020448), a Wellcome Trust Investigator Award in Science (207550), and the Royal Society Wolfson Research Merit Award.

Reprint requests should be sent to Lin Wang, Department of Psychiatry, Massachusetts General Hospital, Harvard Medical School, Building 149, 13th Street, Charlestown, MA 02129, or via e-mail: lwang48@mgh.harvard.edu.

\section{REFERENCES}

Arnal, L. H., \& Giraud, A.-L. (2012). Cortical oscillations and sensory predictions. Trends in Cognitive Sciences, 16, 390-398.

Arnal, L. H., Wyart, V., \& Giraud, A.-L. (2011). Transitions in neural oscillations reflect prediction errors generated in audiovisual speech. Nature Neuroscience, 14, 797-801.

Bastiaansen, M. C., \& Knösche, T. R. (2000). Tangential derivative mapping of axial MEG applied to event-related desynchronization research. Clinical Neurophysiology, 111, 1300-1305.

Bastos, A. M., Vezoli, J., Bosman, C. A., Schoffelen, J.-M., Oostenveld, R., Dowdall, J. R., et al. (2015). Visual areas exert feedforward and feedback influences through distinct frequency channels. Neuron, 85, 390-401.

Bauer, M., Oostenveld, R., Peeters, M., \& Fries, P. (2006). Tactile spatial attention enhances gamma-band activity in somatosensory cortex and reduces low-frequency activity in parieto-occipital areas. Journal of Neuroscience, 26, 490-501.

Bell, A. J., \& Sejnowski, T. J. (1997). The "independent components" of natural scenes are edge filters. Vision Research, 37, 3327-3338.

Bieri, K. W., Bobbitt, K. N., \& Colgin, L. L. (2014). Slow and fast gamma rhythms coordinate different spatial coding modes in hippocampal place cells. Neuron, 82, 670-681.

Brothers, T., Swaab, T. Y., \& Traxler, M. J. (2015). Effects of prediction and contextual support on lexical processing: Prediction takes precedence. Cognition, 136, 135-149.

Clark, A. (2013). Whatever next? Predictive brains, situated agents, and the future of cognitive science. Behavioral and Brain Sciences, 36, 181-204.

Colgin, L. L., Denninger, T., Fyhn, M., Hafting, T., Bonnevie, T., Jensen, O., et al. (2009). Frequency of gamma oscillations routes flow of information in the hippocampus. Nature, $462,353$.

Colgin, L. L., \& Moser, E. I. (2010). Gamma oscillations in the hippocampus. Physiology, 25, 319-329.

DeLong, K. A., Urbach, T. P., \& Kutas, M. (2005). Probabilistic word pre-activation during language comprehension inferred from electrical brain activity. Nature Neuroscience, 8 , 1117-1121.

Dikker, S., \& Pylkkanen, L. (2011). Before the N400: Effects of lexical-semantic violations in visual cortex. Brain and Language, 118, 23-28.

Dikker, S., \& Pylkkänen, L. (2013). Predicting language: MEG evidence for lexical preactivation. Brain and Language, 127, 55-64.

Engel, A. K., Fries, P., \& Singer, W. (2001). Dynamic predictions: Oscillations and synchrony in top-down processing. Nature Reviews Neuroscience, 2, 704-716.

Freunberger, D., \& Roehm, D. (2017). The costs of being certain: Brain potential evidence for linguistic preactivation in sentence processing. Psychophysiology, 54, 824-832.

Fries, P. (2005). A mechanism for cognitive dynamics: Neuronal communication through neuronal coherence. Trends in Cognitive Sciences, 9, 474-480.

Friston, K. (2011). What is optimal about motor control? Neuron, 72, 488-498.

Friston, K. J., Bastos, A. M., Pinotsis, D., \& Litvak, V. (2015). LFP and oscillations-What do they tell us? Current Opinion in Neurobiology, 31, 1-6.

Grisoni, L., Miller, T. M., \& Pulvermüller, F. (2017). Neural correlates of semantic prediction and resolution in sentence processing. Journal of Neuroscience, 37, 4848-4858.

Gruber, T., Müller, M. M., Keil, A., \& Elbert, T. (1999). Selective visual-spatial attention alters induced gamma band responses in the human EEG. Clinical Neurophysiology, 110, 2074-2085.

Hagoort, P., Hald, L., Bastiaansen, M., \& Petersson, K. M. (2004). Integration of word meaning and world knowledge in language comprehension. Science, 304, 438-441.

Hald, L. A., Bastiaansen, M. C., \& Hagoort, P. (2006). EEG theta and gamma responses to semantic violations in online sentence processing. Brain and Language, 96, 90-105.

Hauk, O., Davis, M. H., Ford, M., Pulvermüller, F., \& MarslenWilson, W. D. (2006). The time course of visual word recognition as revealed by linear regression analysis of ERP data. Neuroimage, 30, 1383-1400.

Herrmann, C. S., Lenz, D., Junge, S., Busch, N. A., \& Maess, B. (2004). Memory-matches evoke human gamma-responses. BMC Neuroscience, 5, 13.

Herrmann, C. S., \& Mecklinger, A. (2001). Gamma activity in human EEG is related to highspeed memory comparisons during object selective attention. Visual Cognition, 8, 593-608. 
Jensen, O., \& Colgin, L. L. (2007). Cross-frequency coupling between neuronal oscillations. Trends in Cognitive Sciences, 11, 267-269.

Jung, T.-P., Makeig, S., Westerfield, M., Townsend, J., Courchesne, E., \& Sejnowski, T. J. (2000). Removal of eye activity artifacts from visual event-related potentials in normal and clinical subjects. Clinical Neurophysiology, 111, 1745-1758.

Kim, A., \& Lai, V. (2012). Rapid interactions between lexical semantic and word form analysis during word recognition in context: Evidence from ERPs. Journal of Cognitive Neuroscience, 24, 1104-1112.

Kuperberg, G. R., \& Jaeger, T. F. (2016). What do we mean by prediction in language comprehension? Language, Cognition and Neuroscience, 31, 32-59.

Kutas, M., \& Federmeier, K. D. (2011). Thirty years and counting Finding meaning in the N400 component of the event-related brain potential (ERP). Annual Review of Psychology, 62, 621-647.

Laszlo, S., \& Federmeier, K. D. (2011). The N400 as a snapshot of interactive processing: Evidence from regression analyses of orthographic neighbor and lexical associate effects. Psychophysiology, 48, 176-186.

Leonard, M. K., Ferjan Ramirez, N., Torres, C., Hatrak, M., Mayberry, R. I., \& Halgren, E. (2013). Neural stages of spoken, written, and signed word processing in beginning second language learners. Frontiers in Human Neuroscience, 7, 322.

León-Cabrera, P., Rodríguez-Fornells, A., \& Morís, J. (2017). Electrophysiological correlates of semantic anticipation during speech comprehension. Neuropsychologia, 99, 326-334.

Lewis, A. G., \& Bastiaansen, M. (2015). A predictive coding framework for rapid neural dynamics during sentence-level language comprehension. Cortex, 68, 155-168.

Lewis, A. G., Wang, L., \& Bastiaansen, M. (2015). Fast oscillatory dynamics during language comprehension: Unification versus maintenance and prediction? Brain and Language, 148, 51-63.

Maess, B., Mamashli, F., Obleser, J., Helle, L., \& Friederici, A. D. (2016). Prediction signatures in the brain: Semantic preactivation during language comprehension. Frontiers in Human Neuroscience, 10, 591.

Maris, E., \& Oostenveld, R. (2007). Nonparametric statistical testing of EEG- and MEG-data. Journal of Neuroscience Methods, 164, 177-190.

Michelmann, S., Bowman, H., \& Hanslmayr, S. (2016). The temporal signature of memories: Identification of a general mechanism for dynamic memory replay in humans. PLoS Biology, 14, e1002528.

Mitra, P. P., \& Pesaran, B. (1999). Analysis of dynamic brain imaging data. Biophysical Journal, 76, 691-708.

Molinaro, N., Barraza, P., \& Carreiras, M. (2013). Long-range neural synchronization supports fast and efficient reading: EEG correlates of processing expected words in sentences. Neuroimage, 72, 120-132.

Monsalve, I. F., Pérez, A., \& Molinaro, N. (2014). Item parameters dissociate between expectation formats: A regression analysis of time-frequency decomposed EEG data. Frontiers in Psychology, 5, 847.

Oostenveld, R., Fries, P., Maris, E., \& Schoffelen, J.-M. (2011). FieldTrip: Open source software for advanced analysis of MEG, EEG, and invasive electrophysiological data. Computational Intelligence and Neuroscience, 2011, 1.

Osipova, D., Takashima, A., Oostenveld, R., Fernández, G., Maris, E., \& Jensen, O. (2006). Theta and gamma oscillations predict encoding and retrieval of declarative memory. Journal of Neuroscience, 26, 7523-7531.

Peña, M., \& Melloni, L. (2012). Brain oscillations during spoken sentence processing. Journal of Cognitive Neuroscience, 24, 1149-1164.

Penolazzi, B., Angrilli, A., \& Job, R. (2009). Gamma EEG activity induced by semantic violation during sentence reading. Neuroscience Letters, 465, 74-78.
Piai, V., Anderson, K. L., Lin, J. J., Dewar, C., Parvizi, J., Dronkers, N. F., et al. (2016). Direct brain recordings reveal hippocampal rhythm underpinnings of language processing. Proceedings of the National Academy of Sciences, U.S.A., 113, 11366-11371.

Piai, V., Roelofs, A., \& Maris, E. (2014). Oscillatory brain responses in spoken word production reflect lexical frequency and sentential constraint. Neuropsychologia, 53, 146-156.

Piai, V., Roelofs, A., Rommers, J., \& Maris, E. (2015). Beta oscillations reflect memory and motor aspects of spoken word production. Human Brain Mapping, 36, 2767-2780.

Polanía, R., Paulus, W., \& Nitsche, M. A. (2012). Noninvasively decoding the contents of visual working memory in the human prefrontal cortex within high-gamma oscillatory patterns. Journal of Cognitive Neuroscience, 24, 304-314.

Rao, R., \& Ballard, D. (1999). Predictive coding in the visual cortex: A functional interpretation of some extra-classical receptive-field effects. Nature Neuroscience, 2, 79-87.

Rommers, J., Dickson, D. S., Norton, J. J., Wlotko, E. W., \& Federmeier, K. D. (2017). Alpha and theta band dynamics related to sentential constraint and word expectancy. Language, Cognition and Neuroscience, 32, 576-589.

Rommers, J., Dijkstra, T., \& Bastiaansen, M. (2013). Contextdependent semantic processing in the human brain: Evidence from idiom comprehension. Journal of Cognitive Neuroscience, 25, 762-776.

Staudigl, T., Vollmar, C., Noachtar, S., \& Hanslmayr, S. (2015). Temporal-pattern similarity analysis reveals the beneficial and detrimental effects of context reinstatement on human memory. Journal of Neuroscience, 35, 5373-5384.

Stolk, A., Todorovic, A., Schoffelen, J.-M., \& Oostenveld, R. (2013). Online and offline tools for head movement compensation in MEG. Neuroimage, 68, 39-48.

Summerfield, C., \& de Lange, F. P. (2014). Expectation in perceptual decision making: Neural and computational mechanisms. Nature Reviews Neuroscience, 15, 745-756.

Tallon-Baudry, C., \& Bertrand, O. (1999). Oscillatory gamma activity in humans and its role in object representation. Trends in Cognitive Sciences, 3, 151-162.

Todorovic, A., van Ede, F., Maris, E., \& de Lange, F. P. (2011). Prior expectation mediates neural adaptation to repeated sounds in the auditory cortex: An MEG study. Journal of Neuroscience, 31, 9118-9123.

Van Berkum, J. J. A., Brown, C. M., Zwitserlood, P., Kooijman, V., \& Hagoort, P. (2005). Anticipating upcoming words in discourse: Evidence from ERPs and reading times. Journal of Experimental Psychology: Learning, Memory, and Cognition, 31, 443-467.

Varela, F., Lachaux, J.-P., Rodriguez, E., \& Martinerie, J. (2001). The brainweb: Phase synchronization and large-scale integration. Nature Review Neuroscience, 2, 229-239.

Wang, L., Hagoort, P., \& Jensen, O. (2018). Language prediction is reflected by coupling between frontal gamma and posterior alpha oscillations. Journal of Cognitive Neuroscience, 30, 432-447.

Wang, L., Jensen, O., Van den Brink, D., Weder, N., Schoffelen, J. M., Magyari, L., et al. (2012). Beta oscillations relate to the N400m during language comprehension. Human Brain Mapping, 33, 2898-2912.

Wang, L., Zhu, Z., \& Bastiaansen, M. (2012). Integration or predictability? A further specification of the functional role of gamma oscillations in language comprehension. Frontiers in Psychology, 3, 187.

Wolff, M. J., Jochim, J., Akyurek, E. G., \& Stokes, M. G. (2017). Dynamic hidden states underlying working-memory-guided behavior. Nature Neuroscience, 20, 864-871.

Zhang, H., Fell, J., Staresina, B. P., Weber, B., Elger, C. E., \& Axmacher, N. (2015). Gamma power reductions accompany stimulus-specific representations of dynamic events. Current Biology, 25, 635-640. 